\title{
Effect of Rhizoma alismatis on the expression of hub genes in the treatment of gastric cancer
}

\author{
Jiubo Fan ${ }^{1}$, Hui Jiang ${ }^{2}$, Li Sun ${ }^{3}$, Qin Zhang ${ }^{4}$, Haiju Liu ${ }^{5}$ \\ ${ }^{1}$ Department of Clinical Laboratory, Xiangyang Central Hospital, Affiliated Hospital of Hubei University of Arts and Science, Xiangyang, China; \\ ${ }^{2}$ Department of Clinical Laboratory, Xiangyang Maternal and Child Health Hospital, Xiangyang, China; ${ }^{3}$ Department of Medical and Technology \\ Laboratory, Medicine of Xiangyang Vocational and Technical College, Xiangyang, China; ${ }^{4}$ Department of Clinical Laboratory, Xiangyang Central \\ Hospital, Affiliated Hospital of Hubei University of Arts and Science, Xiangyang, China; ${ }^{5}$ Department of Outpatient Office, Xiangyang Central \\ Hospital, Affiliated Hospital of Hubei University of Arts and Science, Xiangyang, China \\ Contributions: (I) Conception and design: H Liu; (II) Administrative support: J Fan, Q Zhang; (III) Provision of study materials or patients: J Fan, H \\ Jiang; (IV) Collection and assembly of data: L Sun; (V) Data analysis and interpretation: J Fan, Q Zhang; (VI) Manuscript writing: All authors; (VII) \\ Final approval of manuscript: All authors. \\ Correspondence to: Haiju Liu. Department of Outpatient Office, Xiangyang Central Hospital, Affiliated Hospital of Hubei University of Arts and \\ Science, Xiangyang 441021, China. Email: 474092903@qq.com.
}

Background: The aim of this study was to predict the target genes and pathways of Rhizoma alismatis (RA) in the treatment of gastric cancer (GC) by an bioinformatics analysis.

Methods: The Traditional Chinese Medicine System Pharmacology database was used to obtain the chemical components and target genes of RA. GC-related genes were downloaded from GeneCard website. GO and KEGG enrichment analyses were used to detect the potential mechanisms of RA targets. Hub genes were identified by protein-protein interaction (PPI) network and then verified by quantitative real-time polymerase chain reaction (qRT-PCR) analysis.

Results: Our analyses identified 34 target genes that contribute to the development of GC. GO analysis showed that the biological functions of the target genes mainly included activation of receptors, including the nuclear receptor, steroid hormone receptor, acetylcholine receptor, G-protein coupled serotonin receptor, serotonin receptor and others. According to KEGG analysis, we found that insulin resistance, galactose metabolism, adipocytokine signaling pathway, breast cancer pathway, and cholinergic synapse were the top 5 pathways involving RA target genes. qRT-PCR and immunohistochemical analysis indicated that RA had significant effects on the expression of hub genes, including MYC, CASP3, SP1, MAPK8, PPARG, FOS, and SLC2A1.

Conclusions: Our study revealed the multi-component, multi-target, and multi-mechanisms of RA on GC, which suggest novel therapeutics for GC.

Keywords: Gastric cancer (GC); traditional Chinese medicine (TCM); pharmacological network

Submitted Jun 15, 2021. Accepted for publication Sep 07, 2021.

doi: $10.21037 /$ tcr-21-1041

View this article at: https://dx.doi.org/10.21037/tcr-21-1041

\section{Introduction}

Although the treatment of gastric cancer (GC) has made good progress in the past few decades, leading to a significant decline in morbidity and mortality, it) is still the fifth most common malignant tumor in the world and represents a heavy medical burden especially in Asia (1).

In China, the incidence of GC ranks second among men and third among women. GC is also the second largest inducer of cancer-related death in men and women (2). 
There are many reasons for the aberrant overall survival of $\mathrm{GC}$, but main reason is that most patients are diagnosed with advanced disease or even metastasis, so they lose the opportunity to undergo radical resection. Therefore, the development of more advanced diagnostics and precision treatment is a promising direction for cancer treatment $(3,4)$.

Recently, traditional Chinese medicine (TCM) has been reported widely used in the treatment of various diseases, especially for cancers (5). Some TCM has been verified as playing an important role in inhibiting the invasion and migration of many tumors, therefore providing novel methods of cancer treatment. For example, Huangqi has shown excellent effects in preventing cancer progression (6). Despite the advances in cancer treatment, side effects of chemotherapy and radiotherapy still remain, leading to great agony for patients. Chinese herbal medicines are widely prescribed and confirmed to be safe and effective in annihilating many tumors without severe side effects (7). Thus, it would be helpful to explore more effective TCM in the treatment of cancers and to understand the mechanisms of these herbs.

Zexie, also known as Rhizoma alismatis (RA), is a widely used natural medicine with long history in China. As a well-known traditional herb, RA is a crucial component of many prescriptions and has been extensively used for a variety of diseases, including nephropathy, edema, diabetes, inflammation, and cancers (8). However, although RA is widely used for a wide range of diseases, the molecular mechanisms of action in GC are still unclear. Systems biology can be used to understand the role of RA and its components in cancer progression and treatment from the perspective via the method of network pharmacology.

Network pharmacology is a new approach that analyzes the network of biological systems and selects specific signal nodes for multi-target drug molecular design. Because its design concept is consistent with the treatment principles of TCM $(9,10)$, network pharmacology is being widely used in TCM research. For example, eight therapeutic targets of Yin-Huang-Qing-Fei have been identified and associated with the inflammatory process in the progression of asthma (11). In this study, we aimed to explore the putative therapeutic targets of RA using network pharmacology to facilitate an in-depth understanding of the mechanisms of RA in the treatment of GC. We present the following article in accordance with the MDAR reporting checklist (available at https:// dx.doi.org/10.21037/tcr-21-1041).

\section{Methods}

\section{Prediction of drug targets and GC-related genes}

We used the Traditional Chinese Medicine System Pharmacology (TCMSP) database to identify the chemical ingredients and targets of RA. The Genecard website (https://www.genecards.org/) was used to find relevant genes contributing to GC. We then intersected the GCrelated genes and RA target genes by $\mathrm{R}$ software.

\section{Functional analysis of $R A$ target genes}

Related functions and pathways were obtained by GO and KEGG enrichment analyses using the "clusterProfiler" package of $\mathrm{R}$ software. The screening criterion was $\mathrm{q}<0.05$ and $\mathrm{P}<0.05$. Cytoscape 3.7.2 was used to construct the drugcompound-target-disease network and the protein-protein interaction (PPI) network.

\section{PPI network construction}

The STRING database was used to identify potential interactions among the key module genes. The networks (PPIs) with a confidence score $\geq 0.4$ were reserved and further imported to Cytoscape (v3.7.2) for constructing the PPI network of key module genes. In order to detect hub clustering modules in the PPI network, we performed module analysis utilizing the "cytoHubba" app with the MCC method in Cytoscape. The Proteinatlas database (https://www.proteinatlas.org/) was used for detection of hub genes' expression in GC tissue.

\section{Quantitative real-time polymerase chain reaction (qRT- PCR)}

GC cells were extracted with Trizol (Invitrogen, Grand Island, NY, USA) to extract total RNA. According to the manufacturer's instructions, cDNA was synthesized using PrimeScript RT kit (TaKaRa Bio, Shiga, Japan). Real-time PCR was performed with a Roche Light Cycler 480 (Roche) using SYBR Green PCR Master Mix (TaKaRa Bio). The primer sequences are listed in Table 1. Each measurement was repeated three times, and the results were normalized by the expression of the GADPH gene. The fold change from the average value was determined by $2^{-\Delta \Delta \mathrm{Ct}}$. 
Table 1 Sequence of PCR primers used in this study

\begin{tabular}{lcc}
\hline Gene & Forward primer sequence $\left(5^{\prime}->3^{\prime}\right)$ & Reverse primer sequence (5' $\left.->3^{\prime}\right)$ \\
\hline MYC & GGCTCCTGGCAAAGGTCA & CTGCGTAGTTGTGCTGATGT \\
CASP3 & CATGGAAGCGATTCAATGGACT & CTGTACCAGACCGAGATGTCA \\
SP1 & TGGCAGCAGTACCAATGGC & CCAGGTAGTCCTGTCAGAACTT \\
MAPK8 & TGTGTGGAATCAAGCACCTTC & AGGCGTCATCATAAAACTCGTTC \\
PPARG & GGGATCAGCTCCGTGGATCT & TGCACTTTGGTACTCTTGAAGTT \\
NR3C1 & ACAGCATCCCTTTCTCAACAG & AGATCCTTGGCACCTATTCCAAT \\
SREBF1 & ACAGTGACTTCCCTGGCCTAT & GCATGGACGGGTACATCTTCAA \\
FOS & CCGGGGATAGCCTCTCTTACT & CCAGGTCCGTGCAGAAGTC \\
SLC2A1 & GGCCAAGAGTGTGCTAAAGAA & ACAGCGTTGATGCCAGACAG \\
GADPH & ACAACTTGGTATCGTGGAAGG & GCCATCACGCCACAGTTC \\
\hline
\end{tabular}

\section{Cell culture}

AGS, SNU216 GC cells were purchased from the Cancer Cell Repository (Shanghai Cell Bank, Shanghai, China). All cells were cultured in DMEM supplement with $10 \%$ of $(\mathrm{v} / \mathrm{v})$ heat-inactivated fetal bovine serum (Gibco BRL, Gaithersburg, MD, USA) and antibiotics $(100 \mathrm{U} / \mathrm{mL}$ penicillin and $100 \mathrm{U} / \mathrm{mL}$ streptomycin; Hyclone Laboratories. Inc., Logan, UT, USA) at $37^{\circ} \mathrm{C}$ in a humidified atmosphere of $5 \% \mathrm{CO}_{2}$.

RA was extracted and boiled twice with distilled water for $3 \mathrm{~h}$ and the extracts were centrifuged for $10 \mathrm{~min}$ at 10,000 rpm $(8,363 \mathrm{~g})$ at $4{ }^{\circ} \mathrm{C}$. The supernatant was lyophilized and stored at $-20{ }^{\circ} \mathrm{C}$ for assays. GC cells were induced by RA at a concentration of $4.5 \mathrm{mg} / \mathrm{mL}$ for $3-5$ days.

\section{CCK-8 assay}

Cell Counting Kit-8 (CCK-8 assay kit, Dojindo, Japan) was conducted to measure cell proliferation capacity at a density of $5 \times 10^{3}$ cells in each well of 96 -well plates. After incubation for $0,12,24,48,72 \mathrm{~h}, 10 \mu \mathrm{L}$ of CCK-8 solution was added into each well. Absorbance was examined utilizing MRX II microplate reader (Dynex Technologies, Chantilly, VA, USA) at $450 \mathrm{~nm}$.

\section{Statistical analysis}

SPSS 23 software (SPSS, Chicago, IL, USA) was used to analyze statistical data. Data are expressed as mean \pm SD. Student's $t$ test was used to compare the variation of two groups in terms of mean $\pm \mathrm{SD}$. $\mathrm{P}<0.05$ was considered to indicate a statistically significance difference.

\section{Results}

\section{Analysis of $R A$ ingredients and prediction of drug targets}

From the TCMSP database we identified 26 constituents and 37 relevant target genes of RA.

\section{Prediction of GC targets}

By using the keyword "gastric cancer" in the Genecard website, we found 11,843 relevant genes that might contribute to GC. When we intersected the GC-related genes and RA target genes by $\mathrm{R}$ software, 34 shared genes were finally selected (Figure 1A).

\section{Network of $R A$ and its targets in $G C$}

To better understand the interaction of RA and its target genes, we used Cytoscape to establish a drug-compoundtarget-disease network of RA (Figure $1 B$ ). The PPIs of whole shared genes were constructed by the STRING database (Figure $2 A$ ). The downloaded data were visualized by Cytoscape software and analyzed by the cytoHubba application to identify the hub genes, which were MYC, CASP3, SP1, MAPK8, PPARG, NR3C1, SREBF1, FOS, SLC2A1, and PGR (Figure 2B). The bar plot in Figure $2 C$ shows the gene connections with other key genes. After intersecting the two groups of genes, the results indicated 
A

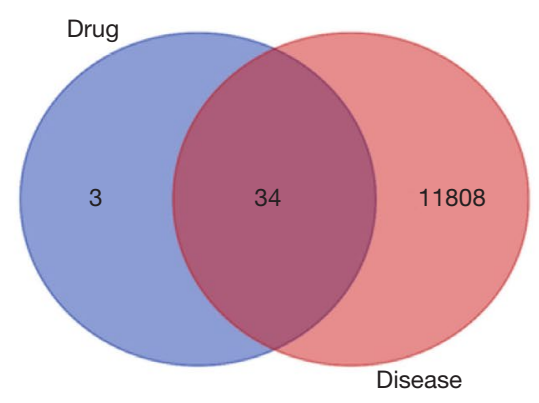

B

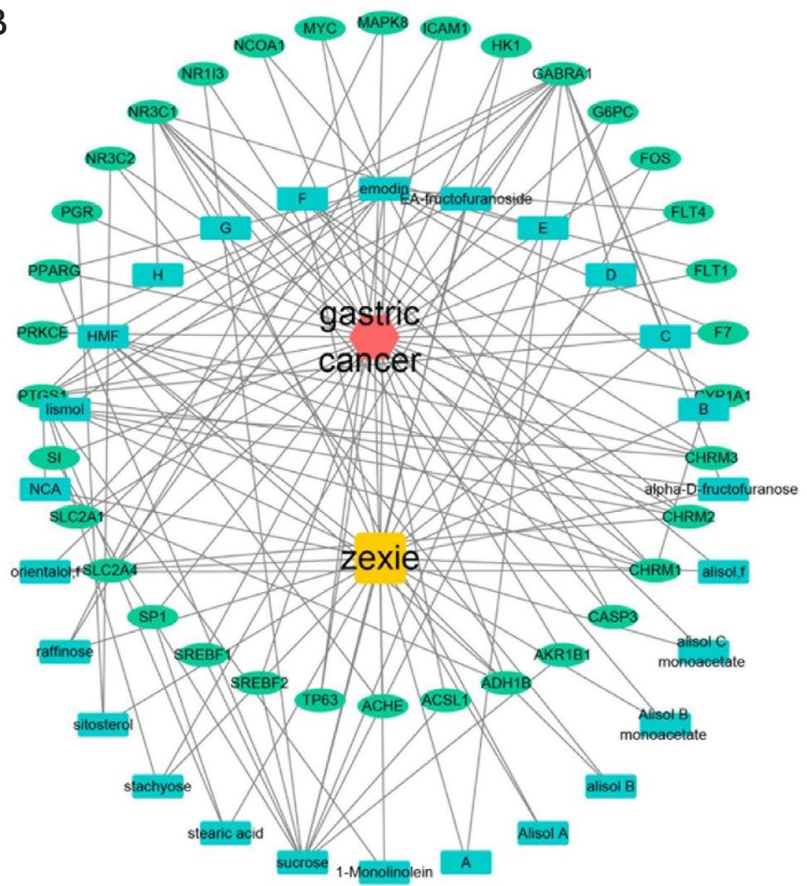

Figure 1 Interaction between drug targets and gastric cancer-related genes. (A) Venn diagram of disease and drug targets. (B) Drugcompound-target-disease network map.

that MYC, CASP3, SP1, MAPK8, PPARG, NR3C1, SREBF1, FOS, and SLC2A1 were the common key genes. To investigate whether RA inhibited the proliferation of GC cells in vitro, we performed CCK8 assay and found that RA significantly abated proliferation of SNU216 and AGS GC cells (Figure $3 A$ ). The qRT-PCR analysis revealed that the expression levels of MYC, SP1, MAPK8, PPARG, FOS, and SLC2A1 were significantly downregulated in GC cells after treatment with RA, while CASP3 and SREBF1 were upregulated (Figure 3B). Immunohistochemical results from the Proteinatlas database showed that MYC, SP1, MAPK8, PPARG, FOS, NR3C1, and SLC2A1 were overexpressed in GC tissue, while CASP3 and SREBF1 showed low levels of expression (Figure 3C).

\section{GO and KEGG analyses}

To further explore the potential molecular mechanism in the common genes contributing to GC, functional enrichment analysis including GO and KEGG was performed. Hub genes were mainly enriched in the activation of many receptors, including nuclear receptor, steroid hormone receptor, acetylcholine receptor, G-protein coupled serotonin receptor, serotonin receptor, neurotransmitter receptor, and others. As well, direct ligand-regulated sequence-specific DNA binding, DNAbinding transcription activator activity, E-box binding, and core promoter binding were also involved in the target genes. According to the KEGG analysis, we found that insulin resistance, galactose metabolism, adipocytokine signaling pathway, breast cancer pathway, and cholinergic synapse were top pathways involving RA target genes (Figure 4A,4B).

\section{Discussion}

The standard treatment for GC is still surgery combined with chemotherapy (12). However, due to the development of multidisciplinary team management and moleculartargeted therapy, the development of personalized treatment for patients is becoming increasingly important $(13,14)$. Because radical surgery for GC is often accompanied by complications, and high mortality rates, GC has a poor prognosis, and suitable TCM treatments might have beneficial effects. For example, because of its multi-target effect, TCM has become an important source of therapeutic drugs for ulcerative colitis (15).

The relationship between drugs and diseases can be 


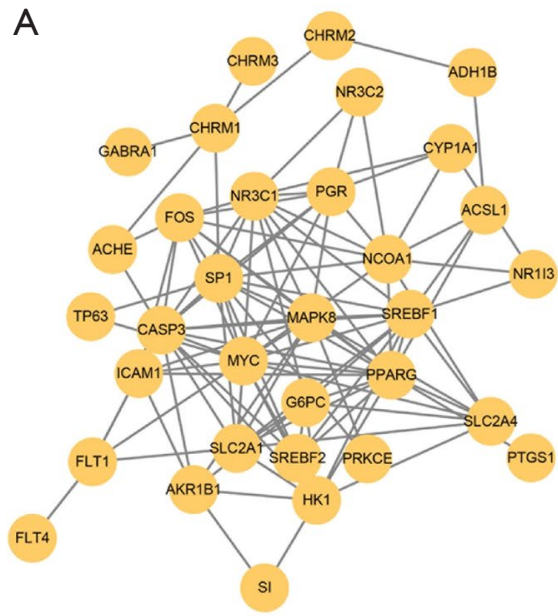

B

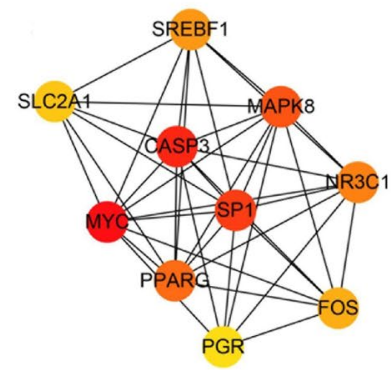

C

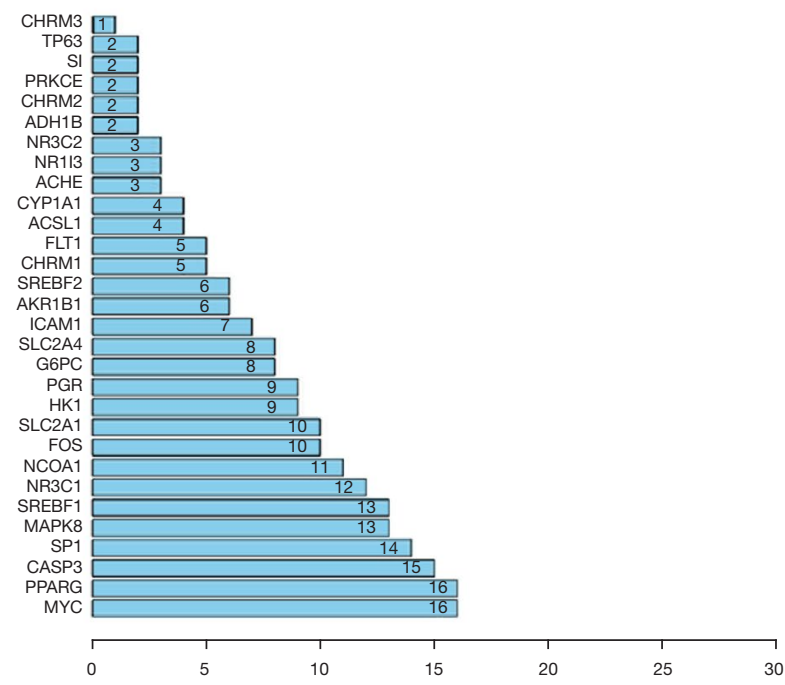

Figure 2 The PPI networks show (A) all common genes and (B) 10 hub genes selected by the "cytohubba" application. (C) Bar plot of the connectivity between genes. PPI, protein-protein interaction

explored from a holistic perspective through network pharmacology, which is a new strategy for TCM research. RA (Zexie), an important traditional Chinese herb, and has a pivotal role in the management of many diseases, including as an analgesic, and as a treatment for dysuria, hyperlipidemia, and cancers (16). However, the role of $\mathrm{RA}$ in facilitating the treatment of GC has been unclear. In our study, we used the TCMSP database to identify 26 chemical components of RA and 37 corresponding gene targets, which were overlapped with 11,843 GC-relevant genes identified in the Genecard website. Finally, 34 genes were selected for further analysis by GO enrichment and KEGG pathway analyses, which found that the target genes were mainly related to the biological process of activation of receptors, including the nuclear receptor, steroid hormone receptor, acetylcholine receptor, G-protein coupled serotonin receptor, serotonin receptor, neurotransmitter receptor, and others. Moreover, several signaling pathways stimulated by RA were involved in GC, including insulin resistance, galactose metabolism, adipocytokine signaling pathway, breast cancer pathway, and cholinergic synapse. Insulin resistance could be regarded as a risk factor for patient survival in early GC (17). Tomizawa et al. found that galactose metabolism regulated proliferation of GC cells in vitro, suggesting an important role of galactose in GC (18). These results also indicated that GC involves abnormalities of many biological processes and signaling pathways.

Subsequently, through PPI network analysis, we identified 10 hub genes and overlapped them with the top 10 genes with highest connectivity. Nine hub genes were finally chosen and verified in GC cell lines in the presence of RA compared with negative control. After treatment with RA, the proliferative ability of GC cells was significantly abated and the expression levels of MYC, SP1, MAPK8, PPARG, FOS, and SLC2A1 were significantly downregulated in the SNU216 and AGS cells, while CASP3 was upregulated. MYC, a crucial protooncogene protein, serves key roles in cellular proliferation, differentiation, transformation and apoptosis in GC (19). $\mathrm{Sp} 1$ is an important transcription factor that could regulate 
A

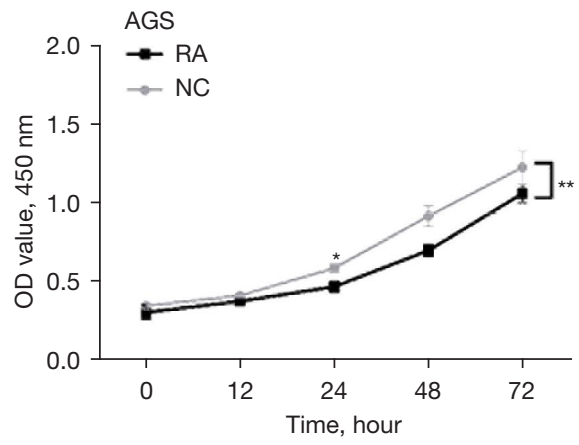

B

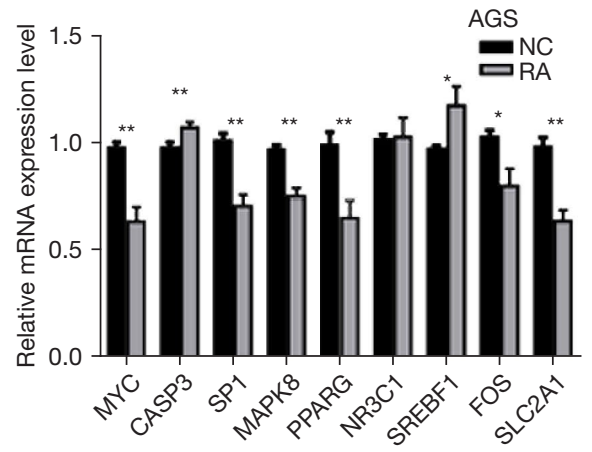

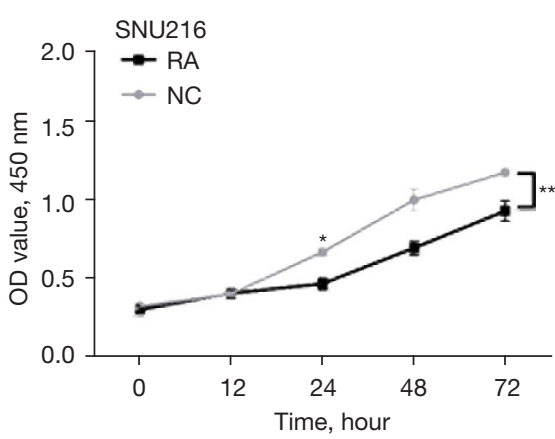

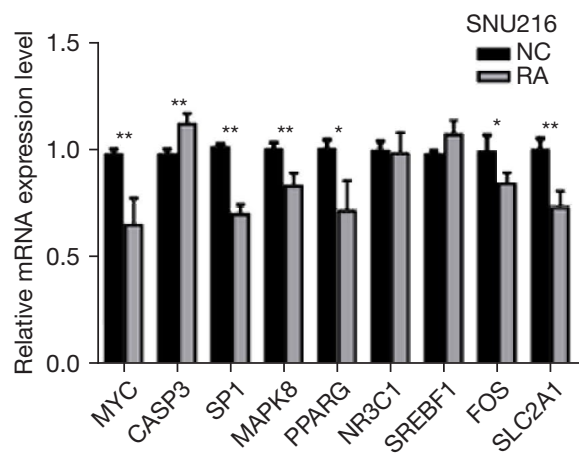

C

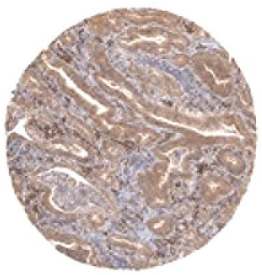

MYC

Staining high Intensity strong

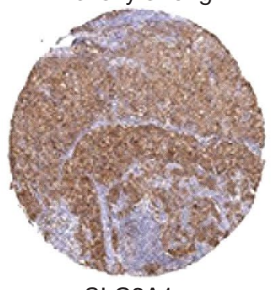

SLC2A1

Staining high

Intensity strong

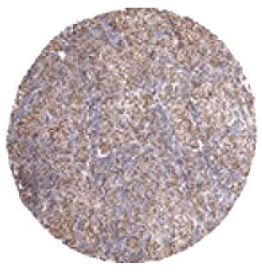

SP1

Staining high Intensity strong

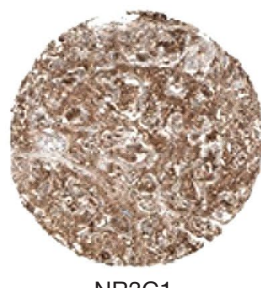

Staining low

Intensity high

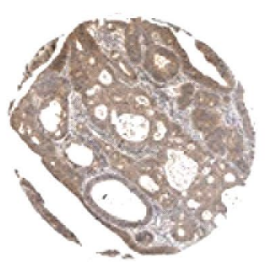

MAKP8

Staining high

Intensity strong

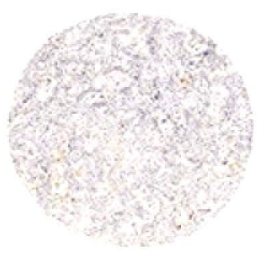

CASP3

Staining low Intensity low

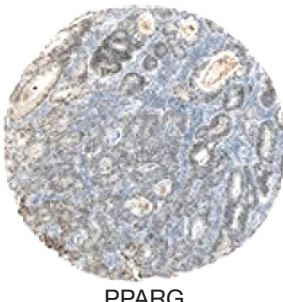

PPARG

Staining high Intensity strong

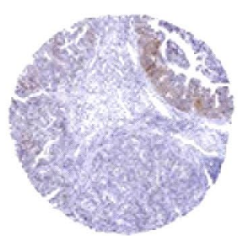

SREBF1

Staining low Intensity weak

Figure 3 Expression levels of hub genes in GC cells and tissue. (A) CCK8 assay shows the proliferation of GC cells was significantly reduced by treatment with RA. (B) qRT-PCR analysis shows mRNA expression levels of each hub gene in the AGS and SNU216 GC cell lines. (C) Immunohistochemical results show the protein expression levels of the hub genes in GC tissue $(100 \times) .{ }^{*}, \mathrm{P}<0.05 ;{ }^{* *}, \mathrm{P}<0.01$. GC, gastric cancer; RA, Rhizoma alismatis; NC, normal control. 

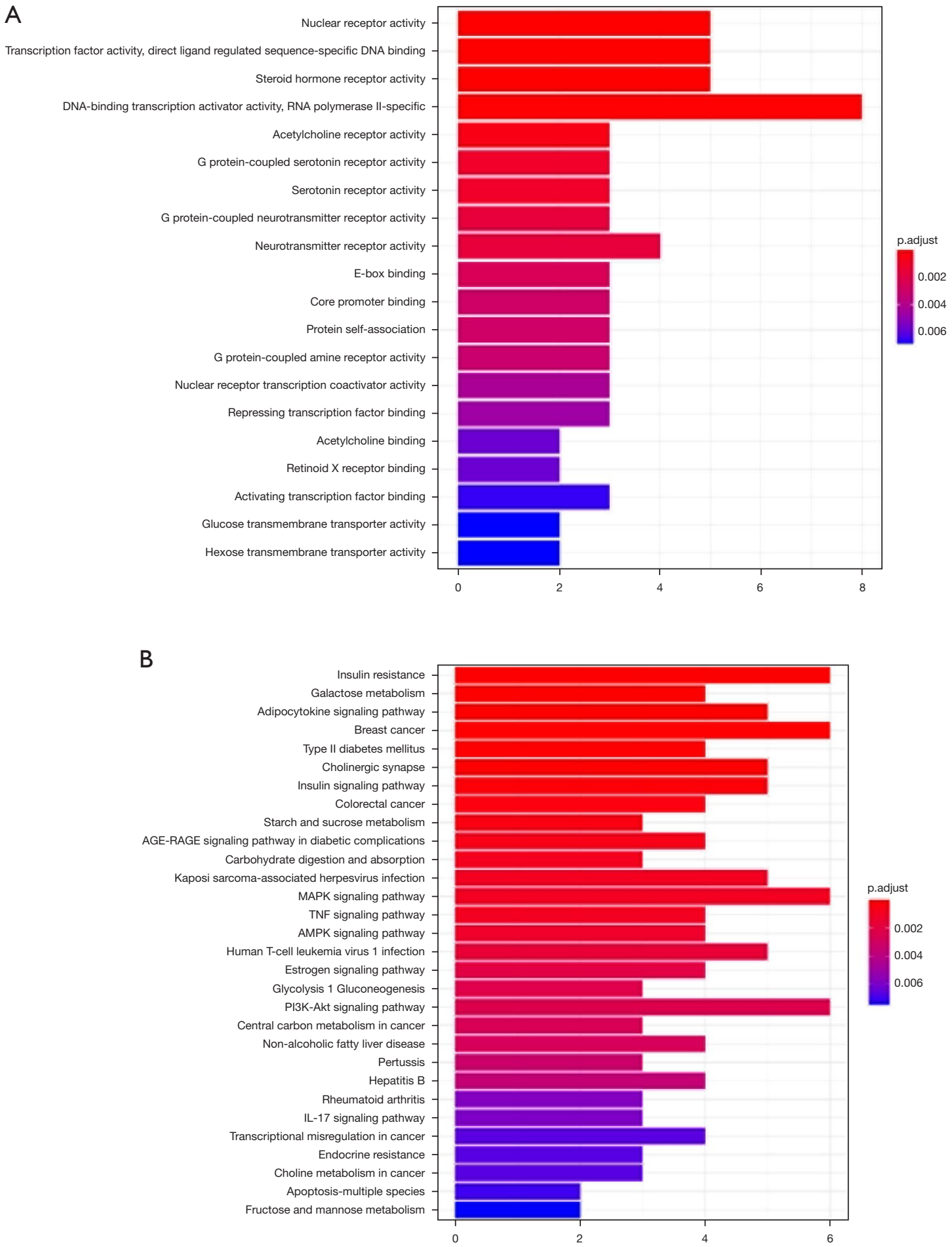

Figure 4 Functional enrichment analyses: (A) GO and (B) KEGG. GO, Gene Ontology; KEGG, Kyoto Encyclopedia of Genes and Genomes. 
multiple cancer-related genes. For instance, SP1 abated the autophagic flux via activation of p62 in GC cells, which regulated progression of GC (20). Therefore, RA has multiple anti-cancer effects by acting on these genes.

Taken together, our study results demonstrate for the first time the pivotal role of RA in the treatment of GC, which could provide novel therapy for GC with little complications. Moreover, we also found the therapeutic targets of RA components and the mechanism of RA in inhibiting GC cell proliferation via pharmacological network analysis. The results provided in-depth understanding of RA and TCM in the treatment of GC and novel directions for cancer treatment.

\section{Study limitations}

Firstly, this study was based on bioinformatics analysis, which needs to be further verified. The RA component related to GC treatment should be confirmed in the future. Moreover, in-depth study of the mechanisms of the effects of the nine hub genes on GC progression is needed.

\section{Conclusions}

The results of network pharmacology analysis showed that RA, a herb used in TCM, can act on multiple targets and exert its effects through multiple signal transduction pathways. These findings clarify the mechanisms of RA as a compound in anti-cancer therapy. The RA targets are currently less studied in cancer, which may provide directions for further targeted therapy.

\section{Acknowledgments}

Funding: This study was supported by Foundation of Xiangyang Medical and Health Technology Plan (2019zd26, 2020YL06).

\section{Footnote}

Reporting Checklist: The authors have completed the MDAR reporting checklist. Available at https://dx.doi. org/10.21037/tcr-21-1041

Conflicts of Interest: All authors have completed the ICMJE uniform disclosure form (available at https://dx.doi. org/10.21037/tcr-21-1041). The authors have no conflicts of interest to declare.
Ethical Statement: The authors are accountable for all aspects of the work in ensuring that questions related to the accuracy or integrity of any part of the work are appropriately investigated and resolved. The study was conducted in accordance with the Declaration of Helsinki (as revised in 2013). Institutional ethical approval and informed consent were waived.

Open Access Statement: This is an Open Access article distributed in accordance with the Creative Commons Attribution-NonCommercial-NoDerivs 4.0 International License (CC BY-NC-ND 4.0), which permits the noncommercial replication and distribution of the article with the strict proviso that no changes or edits are made and the original work is properly cited (including links to both the formal publication through the relevant DOI and the license). See: https://creativecommons.org/licenses/by-nc-nd/4.0/.

\section{References}

1. Ferro A, Peleteiro B, Malvezzi M, et al. Worldwide trends in gastric cancer mortality (1980-2011), with predictions to 2015 , and incidence by subtype. Eur J Cancer 2014;50:1330-44.

2. Chen $W$, Zheng R, Baade PD, et al. Cancer statistics in China, 2015. CA Cancer J Clin 2016;66:115-32.

3. Wu Y, Li Y, Liu J, et al. The diagnostic value of extracellular protein kinase A (ECPKA) in serum for gastric and colorectal cancer. Transl Cancer Res 2020;9:3870-8.

4. Zong L, Abe M, Seto Y, et al. The challenge of screening for early gastric cancer in China. Lancet 2016;388:2606.

5. Wang Y, Fan X, Qu H, et al. Strategies and techniques for multi-component drug design from medicinal herbs and traditional Chinese medicine. Curr Top Med Chem 2012;12:1356-62.

6. Kavandi L, Lee LR, Bokhari AA, et al. The Chinese herbs Scutellaria baicalensis and Fritillaria cirrhosa target $\mathrm{NF \kappa B}$ to inhibit proliferation of ovarian and endometrial cancer cells. Mol Carcinog 2015;54:368-78.

7. Liu J, Pan J, Wang Y, et al. Component analysis of Chinese medicine and advances in fuming-washing therapy for knee osteoarthritis via unsupervised data mining methods. J Tradit Chin Med 2013;33:686-91.

8. Tian T, Chen H, Zhao YY. Traditional uses, phytochemistry, pharmacology, toxicology and quality control of Alisma orientale (Sam.) Juzep: a review. J Ethnopharmacol 2014;158 Pt A:373-87. 
9. Boezio B, Audouze K, Ducrot P, et al. Network-based Approaches in Pharmacology. Mol Inform 2017. doi: 10.1002/minf.201700048.

10. Hao da C, Xiao PG. Network pharmacology: a Rosetta Stone for traditional Chinese medicine. Drug Dev Res 2014;75:299-312.

11. Yu G, Zhang Y, Ren W, et al. Network pharmacologybased identification of key pharmacological pathways of Yin-Huang-Qing-Fei capsule acting on chronic bronchitis. Int J Chron Obstruct Pulmon Dis 2017;12:85-94.

12. Ilson DH. Carcinomatosis in gastric cancer: the potential for cytoreductive surgery and intraperitoneal chemotherapy. Chin Clin Oncol 2020;9:24.

13. Johnston FM, Beckman M. Updates on Management of Gastric Cancer. Curr Oncol Rep 2019;21:67.

14. Stewart C, Chao J, Chen YJ, et al. Multimodality management of locally advanced gastric cancer-the timing and extent of surgery. Transl Gastroenterol Hepatol. 2019;4:42.

15. Chen XL, Wen Y, Wu ZC, et al. Development of a Traditional Chinese Medicine Syndrome-Specific Scale for Ulcerative Colitis: The Large Intestine Dampness-
Heat Syndrome Questionnaire. Evid Based Complement Alternat Med 2018;2018:4039019.

16. Chen DQ, Feng YL, Tian T, et al. Diuretic and antidiuretic activities of fractions of Alismatis rhizoma. J Ethnopharmacol 2014;157:114-8.

17. Kwon HJ, Park MI, Park SJ, et al. Insulin Resistance Is Associated with Early Gastric Cancer: A Prospective Multicenter Case Control Study. Gut Liver 2019;13:154-60.

18. Tomizawa M, Shinozaki F, Motoyoshi Y, et al. Proliferation and motility of hepatocellular, pancreatic and gastric cancer cells grown in a medium without glucose and arginine, but with galactose and ornithine. Oncol Lett 2017;13:1276-80.

19. Gong L, Xia Y, Qian Z, et al. Overexpression of MYC binding protein promotes invasion and migration in gastric cancer. Oncol Lett 2018;15:5243-9.

20. Xu XW, Pan CW, Yang XM, et al. SP1 reduces autophagic flux through activating p62 in gastric cancer cells. Mol Med Rep 2018;17:4633-8.

(English Language Editor: K. Brown)
Cite this article as: Fan J, Jiang H, Sun L, Zhang Q, Liu H. Effect of Rhizoma alismatis on the expression of hub genes in the treatment of gastric cancer. Transl Cancer Res 2021;10(9): 4087-4095. doi: 10.21037/tcr-21-1041 